\title{
Revisión bibliográfica de equipos de trabajo: enfoque cuantitativo, características e identificación de variables que afectan la eficiencia
}

\author{
Literature Review of Work Teams: Quantitative \\ Approach, Characteristics and Identification \\ of Variables Affecting Efficienc
}

\author{
Thomas Leonardo Rangel-Carreño ${ }^{1}$, Ivonne Katherine Lugo-Garzón ${ }^{2} \bowtie$, \\ María Eugenia Calderón ${ }^{3}$
}

\author{
1 Universidad Distrital Francisco José de Caldas, Bogotá, Colombia \\ Correo electrónico: tlrangelc@correo.udistrital.edu.co \\ ${ }^{2}$ Estudiante Ingeniería Industrial, Facultad de Ingeniería, \\ Universidad Distrital Francisco José de Caldas, Bogotá, Colombia \\ Correo electrónico: iklugog@correo.udistrital.edu.co. Autora de correspondencia \\ ${ }^{3}$ Universidad Distrital Francisco José de Caldas,
}

Calle 13 N. ${ }^{0}$ 79c-11, Bogotá, Colombia. Email: iklugog@correo.udistrital.edu.co.

Recibido: 25 de agosto del 2017 Aprobado: 30 de noviembre del 2017 Disponible en línea: 1 de enero del 2018

How to cite this article: T. L. Rangel-Carreño, I. K. Lugo-Garzón y M. E. Calderón, "Revisión bibliográfica de equipos de trabajo: enfoque cuantitativo, características e identificación de variables que afectan la eficiencia”, Revista Ingeniería Solidaria, vol. 14, no. 24, pp. 17, enero 2018. doi: https://doi.org/10.16925/in.v14i24.2164

\section{Resumen}

Introducción: el artículo es el resultado del proyecto de investigación "Modelo matemático para la conformación de equipos de trabajo eficientes", financiado por el Centro de Investigaciones y Desarrollo Científico de la Universidad Distrital Francisco José de Caldas y realizado en el marco de las actividades del Grupo en Gestión Empresarial e Innovación Tecnológica (GEIT) de la misma universidad, entre el 2016 y el 2017.

Objetivo: determinar y conocer las características de los equipos de trabajo y realizar una identificación con el fin de conocer las variables que afectan su eficiencia.

Metodología: se usaron trabajos académicos que estuvieran publicados desde el 2010 en adelante (en las referencias se encuentran unas excepciones). Se utilizaron bases de datos especializadas como Scopus, Science Direct y Redalyc, entre otras. Resultados: se encontraron 57 trabajos entre artículos, monografías y demás que cumplían con el lineamiento de la investigación; además, se encontraron grandes ventajas al usar equipos de trabajo en las organizaciones.

Conclusiones: las variables encontradas son una forma de medir el trabajo en equipo. Las implicaciones de este hallazgo pueden servir para un modelo matemático o alguna serie de indicadores que permita tener contacto con los resultados a nivel de equipo dado que la literatura aboga por el trabajo en equipo pero su cuantificación se queda corta; con este aporte se promueve la cuantificación de las bondades del equipo de trabajo.

Limitaciones: se tienen en cuenta las características más generales y de allí las variables influyentes, por lo que es un punto de partida, pero no una formula general para medir la eficiencia de todos los equipos de trabajo.

Originalidad: el tema de los equipos de trabajo se ha abordado desde una perspectiva cualitativa y se expone cómo este mejoraba los proyectos o resolvía problemas empresariales, sobre todo de manera integral. Se exponían ventajas y características trascendentales, pero no alguna forma de medir aquello que tantas bondades tenía. Con la premisa de que lo que se puede medir es susceptible de mejora, se establece una aproximación a un modelo de eficiencia de los equipos de trabajo como base para futuros trabajos de modelación matemática.

Palabras clave: equipos de trabajo, eficiencia, liderazgo, trabajo en equipo, sinergia, variables 


\title{
Literature Review of Work Teams: Quantitative Approach, Characteristics and Identification of Variables Affecting Efficiency
}

\begin{abstract}
Introduction: The article derives from the research project "Mathematical model for the creation of efficient work teams" funded by the Center for Research and Scientific Development, Universidad Distrital Francisco José de Caldas and conducted as part of the activities of the Business Management and Technological Innovation Group (GET) at the same university, between 2016 and 2017.

Aim: To determine and know the characteristics of work teams and identify the variables affecting their efficiency.

Methods: Research papers published from 2010 onwards were reviewed (the references include some exceptions). Specialized databases such as Scopus, Science Direct and Redalyc, among others, were used.

Results: 57 papers were found among articles, dissertations and other documents that met the research guidelines. We also identified great advantages in using work teams in organizations.

Conclusion: The variables found are a way of measuring teamwork. The implications of this finding can be used in a mathematical model or a series of indicators that allow to appreciate team-level results, given that the literature advocates teamwork but its quantification falls short. This contribution promotes the quantification of the benefits of work teams

Limitations: The most general characteristics are considered and hence the influential variables, which is why it is a starting point but not a universal formula to measure the efficiency of all work teams.

Originality: The topic of work teams has been approached from a qualitative perspective, and it is explained how they improved projects or solved business problems, especially in a comprehensive manner. Advantages and transcendental characteristics were described, but not some way of measuring that which had so many benefits. On the premise that what can be measured is susceptible to improvement, an approach to an efficiency model of work teams is established as a basis for future mathematical modeling works.
\end{abstract}

Keywords: Work teams, efficiency, leadership, teamwork, synergy, variables.

\section{Revisão bibliográfica de equipes de trabalho: abordagem quantitativa, características e identificação de variáveis que afetam a eficiência}

\footnotetext{
Resumo

Introdução: este artigo é resultado do projeto de pesquisa "Modelo matemático para a conformação de equipes de trabalhos eficientes", financiado pelo Centro de Pesquisas e Desenvolvimento Científico da Universidad Distrital Francisco José de Caldas e realizado no âmbito das atividades do Grupo em Gestão Empresarial e Inovação Tecnológica (Geit) da mesma universidade entre 2016 e 2017.

Objetivo: determinar e conhecer as características das equipes de trabalho e realizar uma identificação a fim de conhecer as variáveis que afetam sua eficiência.

Metodologia: utilizaram-se trabalhos acadêmicos publicados de 2010 em diante (encontram-se exceções nas referências). Utilizaram-se também bases de dados especializadas como Scopus, Science Direct, Redalyc, entre outras.

Resultados: encontraram-se 57 trabalhos entre artigos, monografias e demais que cumpriam com o lineamento da pesquisa; além disso, encontraram-se grandes vantagens ao usar equipes de trabalho nas organizações.

Conclusão: as variáveis constatadas são uma forma de medir o trabalho em equipe. As implicações deste achado podem servir para um modelo matemático ou alguma série de indicadores que permita ter contato com os resultados em nível de equipe, tendo em vista que a literatura defende 0 trabalho em equipe, mas sua quantificação é limitada; com essa contribuição, promove-se a quantificação dos benefícios da equipe de trabalho.

Limitações: consideram-se características mais gerais e, disso, as variáveis influentes, razão pela qual é um ponto de partida mas não uma fórmula geral para medir a eficiência de todas as equipes de trabalho.

Originalidade: 0 tema das equipes de trabalho tem sido abordado sob uma perspectiva qualitativa e expõe-se como este melhorava os projetos ou resolvia os problemas empresariais, principalmente de maneira integral. Expuseram-se vantagens e características transcendentais, mas não alguma forma de medir aquilo que tantos benefícios tinha. Com a premissa de que 0 que se pode medir é suscetível de aperfeiçoamento, estabelece-se uma aproximação a um modelo de eficiência das equipes de trabalho como base para futuros trabalhos de modelação matemática.
}

Palavras-chave: equipes de trabalho, eficiência, liderança, trabalho em equipe, sinergia, variáveis. 


\section{Introducción}

La sociedad del conocimiento y las dinámicas empresariales de innovación permanente nos llevan a nuevas formas de trabajo. Hoy en día se hace necesario trascender las estructuras burocráticas y de trabajo individual que son "progresivamente sustituidas por configuraciones descentralizadas, horizontales y en forma de red, cuyos nódulos poseen un elevado grado de autonomía y se encuentran interconectados de modo contingente" $[1$, p. 2], de modo que migran hacia la creación de redes de relaciones humanas; el ser humano tiende a asociarse con otros individuos para perseguir metas específicas a partir de un trabajo colaborativo [2] y, en las organizaciones, la participación de los equipos de trabajo se hace fundamental en el propósito de alcanzar los objetivos de la compañía.

$\mathrm{Al}$ abordar las situaciones de trabajo conjunto se pueden adoptar diversos matices y surgen nuevas formas de interpretar los fenómenos, nuevas competencias y nuevas actitudes, así como nuevos atributos, lo que aparece y se concreta a partir de las relaciones interpersonales que tienen como trasfondo el trabajo compartido [3]. Hoy el reto es "que dos o más trabajadores interactúen entre ellos para conseguir unos determinados resultados. La complejidad de las organizaciones implica trabajar a través de objetivos comunes, en función de unos roles adquiridos o unas funciones predeterminadas" $[4$, p. 3]

No basta con que una empresa posea ventajas competitivas en sus productos o servicios, las personas que la conforman se convierten en un factor determinante de su ventaja competitiva y cada vez más se valoran por su conocimiento, aporte intelectual y de talento en la consecución de metas dentro de la organización [5].

El desarrollo científico y tecnológico, así como las dinámicas de innovación, llevan a una exigencia de trabajo cada vez más flexible, pero de mayor exigencia mental; estas formas de organización nos llevan a la necesidad de establecer formas de gestión y de evaluación del trabajo en equipo y a la búsqueda de su mayor eficiencia. A partir de la revisión y el análisis del trabajo en equipo, su conformación, sus características internas y su relación con la empresa, en este artículo se identifican variables que afectan la capacidad del equipo para lograr sus objetivos trazados con eficiencia, esto es, al usar mejor los recursos asignados.

\section{Metodología}

A partir de la consulta de bases de datos especializadas como Scopus, Science Direct y Redalyc, entre otras, y del análisis de publicaciones académicas, se identifican los mecanismos de conformación, las características y las variables que afectan la eficiencia del trabajo en equipo. La información compilada y analizada se estructura en los siguientes apartados.

\section{Equipos de trabajo (generalidades)}

En los entornos académicos y organizacionales se encuentran problemas, proyectos u objetivos complejos cuya resolución o logro implica una labor intelectual profunda que exige conocimientos y competencias diferentes. Así, las personas se unirán y trabajarán conjuntamente y sus habilidades se complementarán a fin de cumplir eficientemente el trabajo a desarrollar. Desde el punto de vista de la organización se ha profundizado en la pertinencia del trabajo en equipo como fundamento de un accionar más eficaz que permite responder a las tareas corporativas y los retos del entorno [6]; desde el punto de vista del individuo, trabajar en equipo se justifica por razones de autoestima, de seguridad y de logro de beneficios.

\subsection{Equipos de trabajo y grupos de trabajo}

Diferenciar entre equipo y grupo de trabajo permite comprender las formas de trabajar colectivamente, dos conceptos con varias divergencias. El trabajo en grupo es útil cuando se realizan tareas de baja complejidad; bajo esta forma de trabajo, el líder delega a sus miembros las actividades bajo un enfoque tayloriano, de manera que un grupo sería un conjunto de personas que comparten información, ideas y formas para resolver problemas, con tareas rutinarias y mecánicas [7] que podrían llevarlas a cabo personas que trabajan individualmente; los integrantes están más preocupados por el cumplimiento de sus funciones y el logro de sus propios objetivos, y cuentan con escaso grado de incertidumbre y de toma de decisiones. Cuando se trabaja en grupo las personas son responsables de su área de competencia y su compromiso es solo con sus propias metas e intereses particulares. Se 
puede decir que la sola interacción de sus integrantes no asegura la conformación y consolidación de un equipo [7].

En la medida en que las tareas se hacen más complejas y se necesite de la colaboración y aporte de todos sus miembros, de un liderazgo compartido, la dinámica de grupo debe pasar a una de trabajo en equipo. El trabajo en equipo ha superado al trabajo individual en todo tipo de organizaciones [8] pues trae como ventajas que, además de alcanzar los objetivos, se crean sinergias y se comparten experiencias, se aumenta el compromiso y la responsabilidad de los miembros, se favorece la coordinación organizacional, se reducen costos de supervisión para la empresa y se aumenta la eficiencia laboral y la calidad de productos o servicios [9].

Para muchos autores "el equipo" posee mayores connotaciones que el "grupo": "Formar un buen equipo de trabajo no depende únicamente de las habilidades cognitivas de los integrantes, sino también depende directamente de algunas características personales tales como habilidades sociales y tipos de personalidad" [10, p. 7]. En el contexto organizacional, los equipos de trabajo son colectivos que existen para realizar tareas relevantes con un grado de interdependencia [11]; dado que se generan interdependencias entre miembros del equipo y tareas, emerge la posibilidad de ejercer diferentes roles en diferentes momentos del proyecto, entre estos el liderazgo:

Un equipo es un reducido número de personas con habilidades complementarias que están comprometidas con un objetivo común de cuyo cumplimiento son mutuamente responsables. Los miembros individuales interactúan entre sí y con el líder del equipo para conseguir su objetivo común [12, p. 71].

Por su parte, Blanco [13] optó por la definición realizada por Kozlowski y Bell, quienes definen el trabajo en equipo como la colectividad de los integrantes para la realización de las tareas, el hecho de compartir metas u objetivos en común a cumplir, la existencia de una interacción positiva entre sus miembros, poseer interdependencia de las tareas y labores a realizar, así como estar organizados en un contexto organizacional que delimite e imponga fronteras para el equipo y se den intercambios con otras unidades de la organización.
En el trabajo en equipo las habilidades de cada miembro se complementan: "Los equipos se conforman por procesos que luego van generando responsabilidades dentro de éste y en dado caso el liderazgo al interior se comparte" [14, p. 12], y existe un líder que motiva a sus colaboradores y reconoce el trabajo bien hecho de cada uno [15]. En las organizaciones, el trabajo en equipo supera al trabajo individual cuando la tarea a realizar es compleja y se necesita del conocimiento, las habilidades, la creatividad y el compromiso del conjunto para alcanzar el objetivo [16]. Se puede decir que la principal diferencia de los equipos con los grupos de trabajo se encuentra en el nivel de interdependencia de los miembros y de tareas, lo que abre espacios para la cooperación y el aprendizaje e incrementa el compromiso organizacional [13].

Un equipo sería entonces dos o más individuos que interactúen socialmente (cara a cara o de forma virtual); posean uno o más objetivos comunes y se han reunido para desarrollar una tarea organizacional relevante; demuestren interdependencia con respecto a la carga de trabajo, los objetivos y los resultados; tengan diferentes roles y responsabilidades; y se encuentren inmersos en un sistema organizacional [17].

Estrada [7] menciona que los equipos de trabajo tienen objetivos primordiales:

- Generar ideas, identificar causas y generar soluciones a una determinada situación.

- Planear acciones, identificar la secuencia de actividades que permitan alcanzar los objetivos propuestos.

- Afianzar la sinergia para aportar desde los conocimientos y experiencias de cada uno en el logro de los objetivos comunes, de manera que se obtengan resultados superiores a los que se obtendrían individualmente.

En la tabla 1 se muestran las diferencias más marcadas entre el trabajo en grupo y en equipo, así como en el subsiguiente listado, se muestra cómo se podría identificar un equipo a grandes rasgos.

Para saber que existe un equipo de trabajo se logra:

- Llegar a establecer unos objetivos en común.

- Exista un reparto de tareas de acuerdo con las capacidades y cualidades de los miembros componentes. 
- Exista un cierto bienestar entre los miembros.

- Hay alguien que dirija o coordine el trabajo.

- Exista responsabilidad personal en cada uno de los miembros componentes.

Tabla 1. Principales diferencias entre grupos de trabajo y equipos

\begin{tabular}{ll}
\hline \multicolumn{1}{c}{ Grupos } & \multicolumn{1}{c}{ Equipos } \\
\hline $\begin{array}{l}\text { Conjunto de personas que } \\
\text { no tienen un objetivo en } \\
\text { común. }\end{array}$ & $\begin{array}{l}\text { Conjuntos de personas con } \\
\text { un objetivo en mente. }\end{array}$ \\
$\begin{array}{l}\text { Las personas pueden tener } \\
\text { los mismos intereses. }\end{array}$ & $\begin{array}{l}\text { Las personas tienen y } \\
\text { comparten los mismos } \\
\text { intereses. }\end{array}$ \\
$\begin{array}{l}\text { Las personas deciden de } \\
\text { forma individual. }\end{array}$ & $\begin{array}{l}\text { Las personas deciden de } \\
\text { forma conjunta. }\end{array}$ \\
$\begin{array}{l}\text { Las personas actúan de } \\
\text { manera individual. }\end{array}$ & $\begin{array}{l}\text { Las personas actúan de } \\
\text { manera conjunta. }\end{array}$ \\
$\begin{array}{l}\text { No hay interconexión ni } \\
\text { intercambio de ideas. }\end{array}$ & $\begin{array}{l}\text { Tienen una fuerte interco- } \\
\text { nexión e intercambio de }\end{array}$ \\
$\begin{array}{l}\text { Resultan en una suma de } \\
\text { esfuerzos de las personas. }\end{array}$ & $\begin{array}{l}\text { Resultan en la multiplica- } \\
\text { ción de los esfuerzos de las } \\
\text { personas. }\end{array}$ \\
$\begin{array}{l}\text { No hay interacción emo- } \\
\text { cional o afectiva. }\end{array}$ & $\begin{array}{l}\text { Existen una fuerte interac- } \\
\text { ción emocional y afectiva. }\end{array}$ \\
\hline
\end{tabular}

Fuente: [18, p. 233]

Se pueden reconocer equipos de trabajo si tienen las siguientes características, como se muestran en [19]:

- Los equipos constan normalmente de 5 a 15 miembros.

- Creación expresa para la búsqueda de un objetivo en concreto.

- Coordinación.

- Tareas definidas y complementarias.

- Buenas relaciones personales entre integrantes.

"Los equipos son una parte formal y reconocida de una organización y representan una desviación respecto al diseño jerárquico tradicional. En los equipos se permite compartir autoridad, responsabilidades y el dar cuenta en las decisiones y resultados mediante la participación y el compromiso" [19].

Según la literatura, los equipos de trabajo pueden tener varios enfoques:
- Equipos centrados en las personas (equipo de trabajo). En estos equipos se privilegian las personas sobre la tarea y los resultados.

- Equipos centrados en la tarea (trabajo en equipo). El interés del equipo se centra en los procesos que se realizan en la tarea.

- Equipos centrados en los resultados (trabajo de equipo). El interés de los integrantes del equipo se centra en la obtención de los resultados.

Finalmente, vale la pena resaltar que trabajar en los equipos con la gestión de la información genera un enfoque sistémico en los equipos de trabajo [20].

\subsection{Equipos de trabajo. Ventajas y retos}

Los equipos aportan diversidad en conocimientos, actitudes, habilidades y experiencias cuya integración permite ofrecer respuestas rápidas, flexibles e innovadoras a los problemas y retos planteados, promoviendo el rendimiento y mejorando la satisfacción de sus integrantes [21]. Un equipo se justifica en razón de que sus resultados son siempre mayores que cuando trabaja cada persona por separado [6].

En los equipos "se garantiza la interacción entre los miembros para cumplir un objetivo común, aprovechando las competencias individuales para un mejor desempeño y responsabilidad colectivas. Se genera interdependencia empleando las fortalezas de cada miembro" [22, p. 1]. Gracias a su capacidad de diálogo e interpretación los equipos pueden ajustar sus enfoques a la nueva información y a los nuevos retos con mayor velocidad, exactitud y eficacia que un individuo aislado con gran cantidad de conexiones organizacionales [23]. La pertenencia a una unidad de trabajo puede ayudar al individuo a satisfacer sus necesidades de afiliación, aumentar su seguridad, facilitar su desarrollo personal y profesional, así como ser una fuente de creatividad [24]. Las transformaciones del trabajo en equipo se ven dentro y fuera de las organizaciones en que se integran, "ello concede un papel primordial a los equipos como forma organizativa. Así, encontramos nuevos escenarios de trabajo, nuevas demandas y nuevas modalidades de equipos" $[25$, p. 5].

En ocasiones los equipos no son mecanismos eficientes de esfuerzos colectivos y se transforman en formas de trabajo que consumen las energía 
física, mental y emocional de sus integrantes, lo que puede ocasionar derroche de esfuerzos para el logro de las metas o que estas no se consigan en absoluto [21]. Un error frecuente es que se tiende a administrar un grupo o un equipo de trabajo de la misma forma, de modo que se obtienen equipos que producen menos que las contribuciones individuales que podrían hacer sus miembros [23], y se asume que los miembros de los equipos ya poseen los conocimientos y habilidades necesarios para trabajar adecuadamente como un equipo.

Los equipos de trabajo son más flexibles que otro tipo de estructuras jerárquicas dentro de la organización, y pueden reunirse y cumplir metas y proyectos conforme a las necesidades lo demanden [26]. La costumbre es buscar individualmente el beneficio personal, por tanto, es común en nuestra sociedad ver que resulta difícil adaptarnos al trabajo en equipo. El trabajo en equipo representa para las organizaciones una herramienta poderosa que permite dar respuesta a las crecientes exigencias externas (productividad, competitividad y tecnología), e internas (trabajadores motivados, productivos y participativos) [27].

\subsection{Equipos de trabajo en la empresa}

En este mundo globalizado las empresas pasan a tener un grado de complejidad enorme y organizan a sus empleados en equipos a fin de llevar a cabo proyectos complejos [28]. Las experiencias se distribuyen a través de diversas personas en la organización, de manera que las diferentes perspectivas y capacidades se pueden encontrar en los individuos para el logro de los propósitos organizacionales: "Los equipos deben contar con los recursos necesarios para realizar su trabajo, esto incluye recursos materiales y humanos así como el apoyo de la organización que permita a sus miembros poner en práctica sus decisiones" [29, p. 79].

\section{Equipos de trabajo eficientes (características)}

Un equipo eficiente se forma cuando personas ponen en común sus conocimientos para crear nuevas ideas y llevar a cabo nuevos proyectos, aunque tengan intereses e ideas diferentes [14], y se organizan en el propósito de resolver problemas o colaborar estrechamente para realizar la tarea asignada [30]. Los equipos pueden ser heterogéneos, y sus miembros tener diferentes formas de pensar, actuar y analizar, u homogéneos y se caracterizan por acoplarse de manera fácil y rápida.

Se plantean como factores en un modelo de eficacia de los equipos de trabajo - visto como un sistema con entradas, transformación y salida- la adaptabilidad, el liderazgo de equipo, la monitorización mutua de rendimiento y las conductas de ayuda o compensatorias [1].

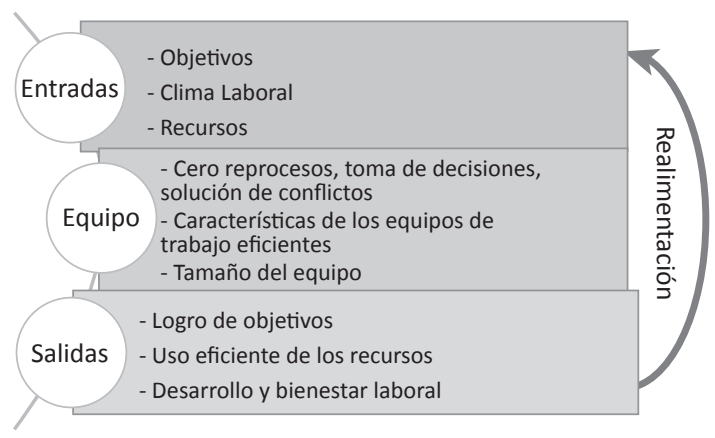

Figura 1. Características de los integrantes en los equipos de trabajo

Fuente: adaptado de [1]

\subsection{Liderazgo}

La labor del líder como guía para el desarrollo efectivo del trabajo en equipo y apoyo para la satisfacción individual de los miembros del equipo debe lograr el compromiso frente a la misión de cada integrante y basarse en la confianza compartida hacia el éxito común [32]. Las organizaciones se enfrentan al reto de ampliar los modelos habituales de liderazgo basados en la autoridad y en el contrato con los subordinados [33]. Un equipo de alto rendimiento no admite el ejercicio de un liderazgo unipersonal y autocrático [14], pues al estar inmerso en la organización y bajo su influencia, comparte y se compromete con los objetivos, acepta adaptarse al cambio, a los retos internos y externos; hoy en día, un líder "inspira confianza y ejerce un estilo de liderazgo transformacional, destacando la importancia de este tipo de liderazgo en el comportamiento" de los equipos [34, p. 162).

El líder motiva, da manejo al equipo, gestiona los recursos asignados [35] y debe [18]:

- Liderar, impulsar y motivar al equipo.

- Evaluar el desempeño del equipo. 
- Recompensar al equipo para reforzar y reconocer su valor.

El líder presenta cuatro rasgos generales que se exponen en la tabla 2 [23].

Tabla 2. Rasgos del líder

\begin{tabular}{c|c}
\hline $\begin{array}{c}\text { Aportar: } \\
\text { Dirección y sentido }\end{array}$ & $\begin{array}{c}\text { Generar y mantener: } \\
\text { Confianza }\end{array}$ \\
\hline $\begin{array}{c}\text { Demostrar: } \\
\text { Propensión a la acción, } \\
\text { tomar riesgos, curiosidad }\end{array}$ & Proporcionar: \\
\hline
\end{tabular}

Fuente: [23]

Como en el caso de la orientación del equipo, el líder puede tener diferentes orientaciones, bien sea hacia las personas - si se preocupa más por la satisfacción de los miembros-, o bien hacia las tareas [36] y actúa como la interfaz entre el equipo y las partes internas y externas, de manera que contribuye al éxito del equipo [37] y permiten a:

Las organizaciones hacer frente al entorno dinámico, a través de formas efectivas para afrontar los cambios en los escenarios sociales, económicos, comerciales y tecnológicos, que no solo les implica la modificación de sus configuraciones estructurales, sino también de sus procesos; es aquí donde la innovación surge como condición para que las empresas se mantengan competitivas y, por ende, la necesidad de que el liderazgo las conduzca hacia esta. [38, p. 7].

\subsubsection{Coordinación}

En relación con la eficiencia del equipo de trabajo, asociada al liderazgo se identifica la variable “Coordinación", la cual corresponde a la gestión de las interdependencias entre tareas, miembros del equipo y recursos, y se encuentra en estrecha dependencia del plan de trabajo del equipo.

\subsubsection{Manejo del conflicto}

Una de las variables asociadas con el liderazgo es el manejo del conflicto y está asociada a la forma como el líder aprovecha las diferencias y divergencias en pensamientos y formas de actuar, y cómo logra una realimentación de los problemas.

Evitar la confrontación de ideas, de datos y de intereses favorece el pensamiento único y la asimetría en la distribución del poder en el equipo. Sin embargo, en "la consideración del conflicto de tareas como fuente generadora de nuevos conocimientos" [39, p. 74] el conflicto constructivo es necesario, ya que las opiniones divergentes enriquecen al grupo, "la diversidad de habilidades y competencias fundamentadas en la confianza y la corresponsabilidad del resultado final garantiza el logro del objetivo del equipo", al tiempo que los líderes deben estimular la toma de decisiones por consenso y fomentar la implicación individual de los miembros del equipo [32, p. 60]: "La integración y no la evitación es el estilo de gestión más eficaz para aminorar los efectos negativos del conflicto relacional" [39, p. 74].

\subsection{Autonomía}

Existe autonomía en un equipo cuando tiene capacidad para tomar decisiones sobre diferentes aspectos de su trabajo: el empoderamiento del equipo genera su autonomía. La autonomía es una característica básica del diseño de la tarea de los equipos que modula los efectos tanto de otras variables antecedentes como de los procesos sobre la eficacia [21]. En un nivel bajo de autonomía el equipo tiene una tarea muy estructurada y definida por la organización, lo que minimiza la necesidad de tomar decisiones colectivas o gestionar los procesos internos. Por el contrario, en un nivel alto de autonomía los miembros del equipo deben tomar numerosas decisiones colectivamente.

Se puede decir que la desconfianza indirecta que demuestran los directivos hacia sus equipos de trabajo al no permitirles tomar decisiones de manera autónoma frena el proceso que pudiera llevarlos al alto desempeño, por lo que se recomienda darles gradualmente autonomía y comenzar por decisiones sencillas hasta otras mayores, siempre y cuando evidencien los resultados obtenidos [40]. El líder cumple una labor importante con la toma de decisiones dado que debe "optimizar al máximo todos los recursos de que disponemos y utilizar todas las herramientas de gestión existentes para facilitar los procesos de análisis y toma de decisiones tanto individuales como grupales" [41], p. 48]. 


\subsection{Comunicación}

Son frecuentes los problemas de comunicación en un equipo de trabajo debido a la personalidad y emotividad de cada uno de sus integrantes. Estas dificultades individuales y grupales las debe identificar y resolver el líder, así como analizar todo oportunamente y de manera responsable [5]. Cuando existe interacción entre todos los miembros del equipo la información es más fluida y se genera mayor participación y satisfacción en sus integrantes:

A partir del circulo surge la estructura completamente conectada, que le da una ventaja: existe una interacción entre todos los integrantes del equipo, por lo cual se puede decir que es la estructura que mayor conviene a una organización que pretende impulsar el desarrollo de los equipos de trabajo debido a que la información es más fluida, hace más partícipes a los integrantes, lo que les genera mayor satisfacción [26, p. 32].

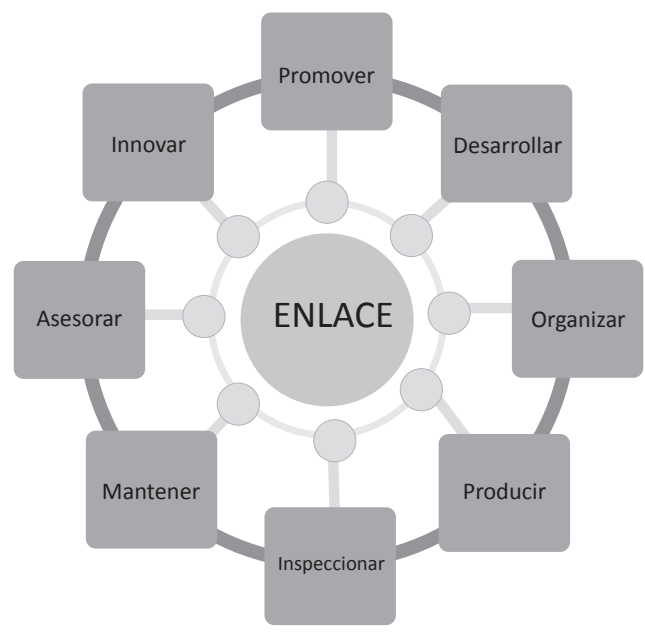

Figura 2 . Nexos e interacción circular de los miembros del equipo

Fuente: adaptado de [26] y [42]

\subsection{Sinergia}

En los equipos de alto desempeño no será el agregado aritmético de las competencias de sus miembros lo que determine el nivel de desempeño; este estará definido por las sinergias que se logren entre los miembros, la especialización que se defina en las tareas, la confianza que se logre entre los sujetos y las habilidades como equipo en torno a la motivación y la comunicación efectiva [32]. Existe una visión global de todos los componentes, estrategias y actividades de una empresa como representantes de esta; en otras palabras, no se trata de la unión simple de las variables, sino que debe observarse la interdependencia de los constructos [6].

Con la sinergia no solo se pueden mejorar los resultados, también se da una dinámica de realimentación en la que se implanta "una cultura empresarial que favorezca el aprendizaje continuo de todos los empleados, de manera que se ponga énfasis en el desarrollo competencial de las personas, para así activar, desarrollar, estimular, o inhibir aquellas competencias que sean necesarias para la organización" [41, p. 12).

\subsubsection{Interdependencia de tareas y miembros}

Se han identificado distintos tipos de interdependencia, y cómo las principales tienen que ver con la tarea y los objetivos. Los estudios centrados sobre la interdependencia de tarea han mostrado su efecto modulador en términos positivos sobre muchos de los efectos que distintos procesos de equipo ejercen sobre los resultados organizacionales [43].

En un equipo los miembros reconocen su interdependencia y entienden que ambos, los objetivos personales y los del equipo, se cumplen mejor con soporte mutuo y asumen una responsabilidad como individuos y como parte del colectivo que integran, y son capaces de modular sus niveles de interdependencia al desempeñar una tarea determinada. La eficiencia se relaciona con la creencia de conseguir el éxito y la capacidad de relacionarse con los miembros del equipo para cumplir las tareas.

\subsubsection{Roles}

Se puede decir que los roles a cumplir dentro de un equipo de trabajo son los que se presentan en la tabla 3.

Los roles determinan las tareas que las personas deben realizar, así como sus actuaciones dentro del equipo que pueden ayudar a mejorar el desempeño $y$, en algunas ocasiones, lo pueden diezmar. Se encuentran roles facilitadores en los cuales las personas se orientan a mantener la armonía del grupo, y de igual forma, roles autoorientados que llevan al conflicto pues las personas que los asumen buscan reconocimiento personal [44]. 
Tabla 3. Roles dentro del equipo

\begin{tabular}{lll}
\hline $\begin{array}{l}\text { Crear soluciones y } \\
\text { resolver problemas }\end{array}$ & $\begin{array}{l}\text { Tener conocimien- } \\
\text { tos específicos } \\
\text { (especialistas) }\end{array}$ & Impulsar \\
\hline Coordinar & Observar y analizar & Cohesionar \\
\hline Implementar & Explorar & Finalizar \\
\hline
\end{tabular}

Fuente: adaptado de [14]

\subsection{Clima laboral}

Un buen ambiente laboral facilita la interrelación entre los miembros del equipo de trabajo, posibilita mejoras individuales y colectivas, mejora la comunicación, genera altos niveles de desempeño, así como favorece la identificación de objetivos comunes y de las cualidades de los diferentes miembros del equipo. De esta manera, se evidencia la estrecha relación que existe entre el clima laboral y el trabajo en equipo, razón por la cual un buen clima laboral se relaciona con el adecuado desempeño de la organización [45].

Un sistema de recompensas centrado en los resultados grupales en lugar de los individuales, un clima caracterizado por un bajo control, alta autonomía, preocupación por el bienestar de los miembros y compromiso por la formación, un entorno no competitivo sino basado en la confianza, las metas compartidas, la seguridad participativa y la controversia constructiva, y un nivel relativamente elevado de incertidumbre ambiental son todas condiciones para que los equipos de trabajo alcancen altos niveles de eficacia [1].

La cooperación en los equipos de trabajo se consigue cuando las personas saben que para ellos será positivo y no un sacrificio, saldrán ganando, se lo reconocerán, conseguirán ayudar a otros sin perjudicarse, les servirá para restituir algún favor anterior y, además, se sentirán bien haciéndolo [46].

\subsection{Sentido prioritario de los objetivos}

La conciliación entre los objetivos individuales y los objetivos del equipo y sus integrantes son aspectos que pueden generar diferencias en la eficiencia del equipo. Los equipos cohesionados tienden a funcionar mejor porque tienen un mayor compromiso por alcanzar los objetivos del equipo, sus miembros son más sensibles a otros y están más dispuestos a fortalecer el espíritu colectivista y el compromiso [7]. La cohesión de un equipo es la fuerza resultante que actúa sobre los miembros de un equipo para permanecer dentro del grupo [13].

\subsection{Tamaño}

En investigaciones realizadas en la década de los ochenta del siglo xx se encontró que la eficacia de los equipos se ve beneficiada conforme aumenta el número de sus integrantes hasta cierto nivel, y se llega a un punto de inflexión que conduce a su decaimiento, por lo tanto, muy pocos o demasiados miembros generan resultados negativos en el rendimiento [13].

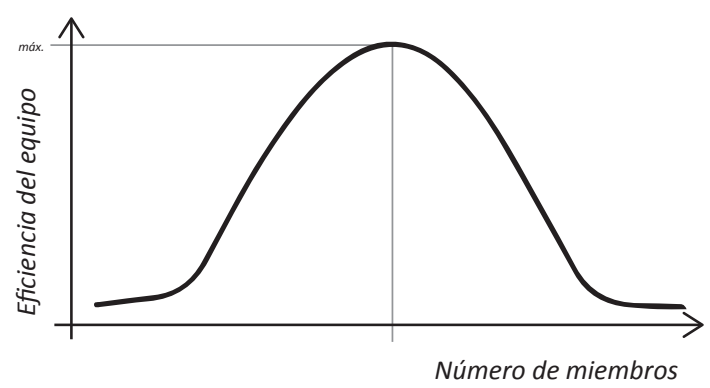

Figura 3. Eficiencia del equipo vs. número de miembros Fuente: elaboración propia

Dos aspectos de la composición del equipo han sido objeto de numerosos estudios: el tamaño (número de personas que forman el equipo), y las características de sus miembros. Las investigaciones indican que el tamaño óptimo depende de ciertas contingencias [21].

\subsection{Aptitudes}

En los equipos de trabajo la aptitud deriva tanto de la capacitación y de la experiencia profesional como del intercambio de conocimientos y de información que fluye dentro de la organización laboral [9].

La efectividad del equipo requiere que sus miembros adquieran un cierto nivel de competencia en su tarea específica asignada [47], además de la adquisición y el dominio de habilidades a nivel individual que es una condición necesaria para servir como un miembro efectivo del equipo. La experiencia hace parte de las aptitudes de los miembros del equipo y afecta directamente su eficiencia. 


\subsubsection{Competencias}

Las competencias de equipo se refieren a los conocimientos, las habilidades y las actitudes que los miembros necesitan para funcionar dentro de este [1], y une un conjunto de conocimientos, procedimientos y actitudes combinados e integrados en el sentido de que el individuo saber hacer y saber estar para el trabajo. Los equipos de trabajo despliegan recursos que les permiten hacer frente a las demandas que el trabajo les exige, y se precisa el desarrollo de recursos personales específicos relacionados con competencias emocionales y sociales, entre ellos la empatía [48].

Las competencias son resultado de un conjunto de aprendizajes acumulativos con énfasis en la aplicación del conocimiento a tareas [11]:

Cuando los equipos mejoran las competencias profesionales y personales de quienes participan en ellos, se da un proceso mediante el cual se convierten en equipos de alto rendimiento, lo que implica que se caractericen por factores críticos de desempeño, que son: objetivos claros, cohesión, confianza, organización y comunicación [6, p. 14].

Es útil conocer en detalle las características personales y profesionales de cada miembro, ya que se pueden utilizar como una ayuda en la integración y la formación de equipos de trabajo [10]. Se identifican como las más críticas habilidades para la efectividad del equipo:

- Habilidades tácticas. La habilidad para determinar o planificar las próximas tareas.

- Habilidades técnicas. La habilidad para realizar las tareas requeridas.

- Habilidades interpersonales. La capacidad de cooperar y comunicarse con otros.

- Habilidades cognitivas. Habilidad para explotar actividades tales como métodos de pensamiento crítico, resolución de problemas, toma de decisiones y su modelo mental [47].

\subsubsection{Conocimientos}

El conocimiento de los miembros del equipo se refiere a la información, la sabiduría y la experiencia necesarias que tienen los miembros a nivel individual en el equipo, lo que les permite completar su tarea dentro de este [47]; la diversidad de conocimientos y habilidades mejora la eficacia de los equipos.

\section{Conformación de equipos de trabajo eficiente}

Construir un buen equipo es coincidir sobre un grupo humano que cuenta con:

- Perfiles profesionales complementarios, y realizan tareas complejas.

- Un clima emocional fundamentalmente cooperativo.

- Una misión y proyecto de ensueño (no utópico), con una dirección y liderazgo de equipo adaptado al perfil de la tarea y de las personas.

En la figura 4 se muestra un modelo para gestionar con éxito equipos de trabajo.

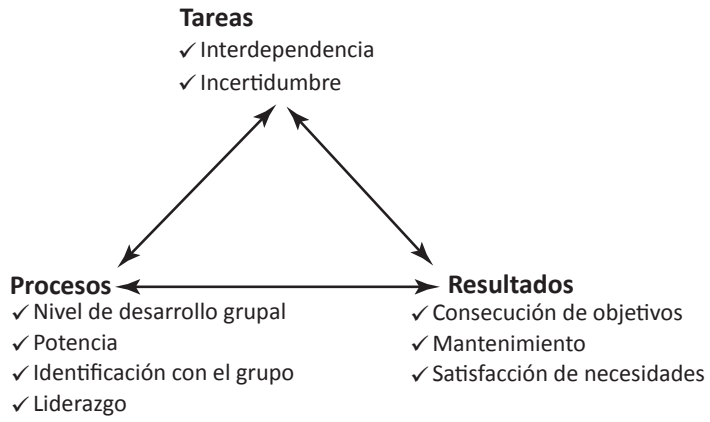

Figura 4. Gestión con éxito de los equipos de trabajo Fuente: tomado de [50]

A fin de formar un equipo hay que cumplir una serie de requisitos:

- Que exista un clima informal, cómodo y relajado, con buena atmósfera de trabajo.

- Las personas deben estar interesadas y cooperando con el éxito del equipo.

- Las tareas y objetivos deben ser aceptados por todos los miembros.

- Cada uno debe ser escuchado y estimulado a expresar todas sus ideas. 
No obstante, se plantea que las

Diferencias en el manejo de los equipos pueden deberse a la propia concepción de equipo que se tiene de los investigadores y organizaciones; ya que no se podía seguir las recomendaciones de la literatura sobre la construcción de equipos, se debe trabajar con agrupaciones de personas que fueran consideradas para la organización como equipos [49, p. 128].

Según el modelo mencionado por Hackman [51], se proporciona una serie de etapas para conformar un equipo de trabajo, tal como se muestran en la tabla 4.

La composición del equipo es una configuración de sus miembros basada en los atributos que influyen en los procesos del equipo y los resultados [35]; la formación de cada miembro [53] y el hecho de que sus habilidades se complementen con las de los demás es importante para realizar las tareas con éxito.

\section{Resultados}

En este apartado se analizan los equipos de trabajo eficientes y se identifican las variables que afectan la eficiencia.

\subsection{Equipos de trabajo eficientes (variables)}

En la figura 5 se presenta el análisis de la eficiencia del trabajo en equipo y su relación con las características de los equipos de trabajo, en el propósito de identificar las variables que explican la eficiencia.

Como se muestra en la figura 5, más allá de la noción tradicional de eficiencia, esta en los equipos de trabajo puede derivarse del análisis de cuatro dimensiones asociadas con el logro de resultados superiores para la empresa en relación con los que se alcanzaría al trabajar individualmente, así:

- Eficiencia tradicional, entendida como el logro del objetivo con calidad y con el menor uso de recursos.

- Resultados adicionales al logro del objetivo del equipo como, por ejemplo, nuevas ideas, nuevos proyectos.

- Bienestar laboral representado en el reconocimiento del individuo y la potenciación de sus capacidades.

- Desarrollo del personal como, por ejemplo, cualificación técnica y desarrollo socioemocional .

Dado lo anterior, tal como se muestra en la figura 5, se identifican como indicadores de eficiencia los siguientes:

- Recurso empleado (i)/Recurso asignado (j) (menor o igual a 1).

- Escalas de valoración individual y colectiva para desarrollo del personal y bienestar laboral.

- Nuevas ideas, proyectos y formas de trabajo.

Tabla 4. Etapas para conformar un equipo

\begin{tabular}{llll}
\hline \multicolumn{1}{c}{ Etapa 1 } & \multicolumn{1}{c}{ Etapa 2 } & \multicolumn{1}{c}{ Etapa 3 } & \multicolumn{1}{c}{ Etapa 4 } \\
\hline Trabajo previo. & $\begin{array}{l}\text { Crear condiciones de } \\
\text { desempeño. }\end{array}$ & $\begin{array}{l}\text { Formar y construir el } \\
\text { equipo. } \\
\text { Diseñar las tareas del grupo. } \\
\text { Ayudar al grupo a establecer } \\
\text { sus límites. } \\
\text { trabajo a realizar. }\end{array}$ & $\begin{array}{l}\text { Prestar asistencia durante la } \\
\text { marcha. }\end{array}$ \\
& $\begin{array}{l}\text { Proporcionar oportunidades } \\
\text { para que el grupo pueda } \\
\text { renegociar sus condiciones } \\
\text { de desempeño. }\end{array}$ \\
$\begin{array}{l}\text { Determinar el nivel de auto- } \\
\text { ridad que el equipo tendrá. }\end{array}$ & $\begin{array}{l}\text { Selección de los miembros } \\
\text { del equipo. }\end{array}$ & $\begin{array}{l}\text { Legitimar y colaborar en el } \\
\text { proceso de redefinición de } \\
\text { tareas. }\end{array}$ & $\begin{array}{l}\text { Otorgar ayuda en el proceso } \\
\text { para promover la sinergia } \\
\text { grupal positiva. }\end{array}$ \\
$\begin{array}{l}\text { Evaluar los costos y bene- } \\
\text { ficios de utilizar un equipo } \\
\text { para realizar el trabajo. }\end{array}$ & $\begin{array}{l}\text { Proveer un soporte con- } \\
\text { textual. } \\
\text { Organizar los recursos } \\
\text { materiales necesarios. }\end{array}$ & de roles y normas grupales. & $\begin{array}{l}\text { Proporcionar oportunidades } \\
\text { para que el grupo aprenda } \\
\text { de sus experiencias. }\end{array}$ \\
\hline
\end{tabular}

Fuente: tomado de [51] 


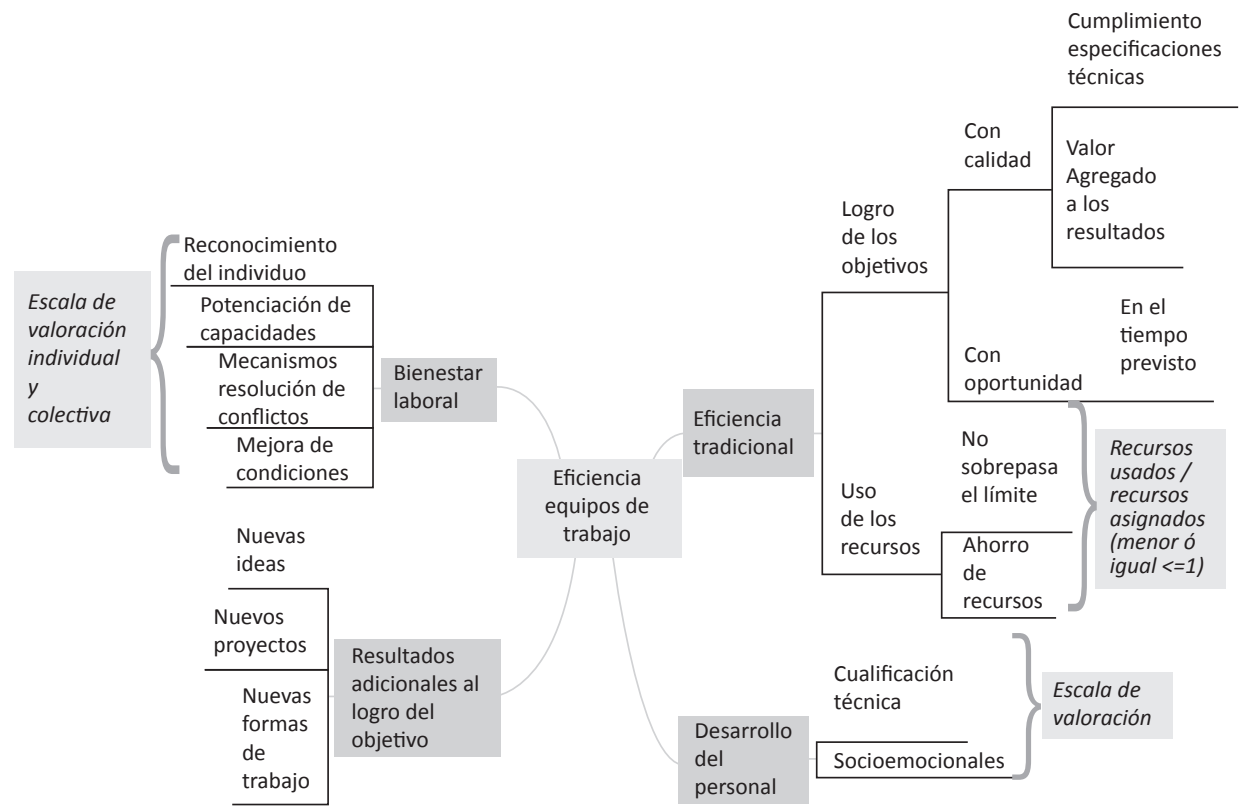

Figura 5. Eficiencia del trabajo en equipo

Fuente: elaboración propia

En la figura 6 se muestra la relación entre las características del trabajo en equipo, las variables identificadas en cada caso y la eficiencia del equipo.

- La coordinación se mide con el indicador de utilización de recursos sobre los recursos asignados; afectará la eficiencia dado que implica el uso de tiempo (y otros recursos, dependiendo de los objetivos del equipo) para lograr los objetivos.

- El impacto de las decisiones en los resultados encontrará cuatro situaciones: 1. La primera es usar menos recursos de los asignados y lograr a su vez el objetivo; 2. La segunda es usar adecuadamente los recursos sin sobrepasarse y lograr el objetivo; 3 . La tercera es usar más recursos de los asignados y así lograr el objetivo; y 4 . Usar más recursos de los asignados sin lograr el objetivo.

- Con la comunicación asertiva la eficiencia se verá afectada, dado que los miembros en la dinámica de equipo deben saber expresar puntos de vista y así estar en capacidad de enviar un mensaje claro y evitar reprocesos, así como comunicar lo correcto en el momento correcto mediante una comunicación oral y no oral. Se verá afectada la eficiencia en la forma en que la comunicación asertiva genere menos reprocesos que incitan a repetir o aclarar lo dicho anteriormente. Será medido por medio de unas auditorías y unos diagnósticos realizados por agentes externos.

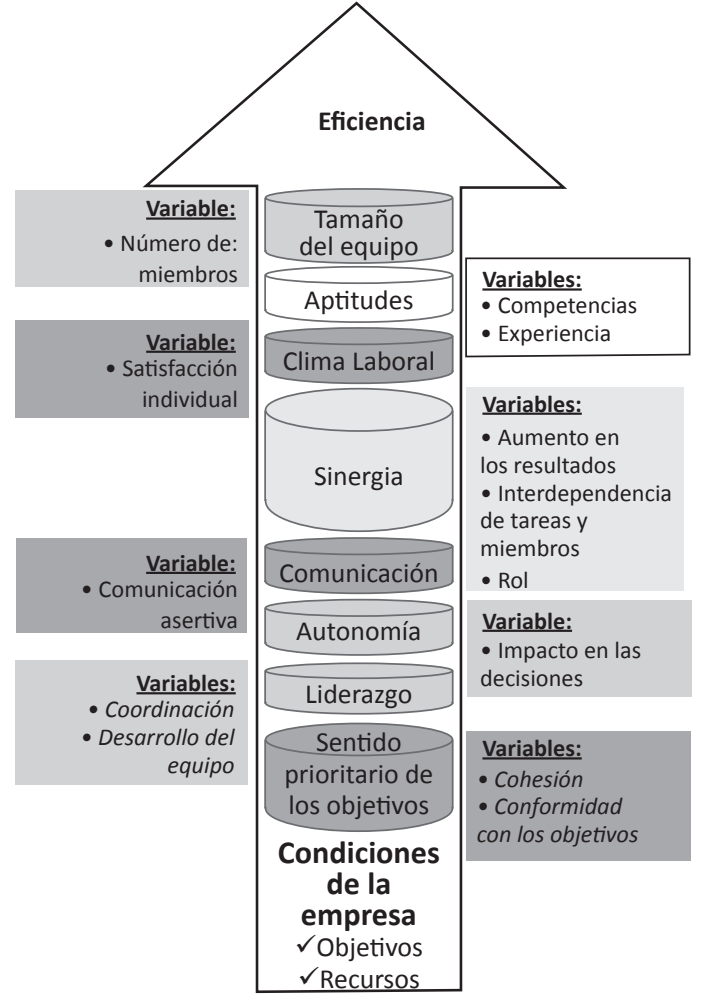

Figura 6. Características y variables del trabajo en equipo que afectan la eficiencia

Fuente: elaboración propia 
- El aumento en los resultados afectará positivamente la eficiencia del equipo, por lo tanto, es una variable en la cual se aprovechan las potencialidades y podrá valorarse mediante la diferencia entre el resultado logrado al alcanzar el objetivo del equipo y el valor agregado sobre este y otros resultados de cualificación de las personas que integran el equipo.

- La interdependencia de tareas y miembros se medirá por la cantidad de tareas que de un individuo dependen; los demás miembros, sobre la cantidad total de actividades presentes en el equipo. El rol se mide mediante los costos de reprocesos. Se comparan con resultados anteriores y se nota un aumento o disminución en la variable.

- La cohesión se mide mediante indicadores de productividad, dado que influye en los esfuerzos de ser mejores día a día y que los empleados con baja cohesión faltan al trabajo o terminan por abandonar al equipo.

- La satisfacción individual se mide mediante una escala al valorar individualmente los sentimientos favorables que se tienen al interior del equipo en una escala de 1 a 5.

- Las aptitudes se valoran a través de un indicador que relacione la culminación de tareas en relación con las tareas totales a realizar. Las competencias se valoran al establecer las habilidades del aspirante sobre las habilidades requeridas (se deben establecer niveles de importancia para las habilidades). La experiencia se mide al realizar una escala, y luego se le asignan valores de acuerdo con el tiempo de experiencia requerido).

- El tamaño del equipo de trabajo es un factor importante: en la medida en que aumenta el tamaño de un equipo, aumenta la cantidad de recursos disponibles (sin embargo, asimismo aumenta la necesidad de coordinación). Se estima que los grupos de trabajo eficiente tienen entre siete y 15 miembros.

\section{Discusión}

La consulta y el análisis bibliográfico muestran que las características de los equipos de trabajo han sido abordadas por diversos autores, así como los mecanismos y enfoques de conformación. Los beneficios que la conformación de equipos de trabajo genera a las empresas han sido expuestos por diversos autores, así como también se ha mostrado que no todos los problemas o retos organizacionales se resuelven con equipos de trabajo.

$\mathrm{Al}$ tener en cuenta que la ingeniería industrial basa sus decisiones en técnicas cuantitativas en la mayor parte de los casos, se encuentra limitación en la identificación de variables, indicadores y relaciones entre las características de los equipos de trabajo y la eficiencia del equipo de trabajo.

Con el análisis presentado en este artículo las variables identificadas y la aproximación a un modelo se brindan pautas que pueden servir de base para una posterior formulación de un modelo de eficiencia del trabajo en equipo.

\section{Conclusiones}

Se evidencia cómo al trabajar bajo una dinámica de equipo se puede aumentar el nivel de eficiencia con respecto a una dinámica más individualista.

Con el análisis realizado se identifican las características y variables que afectan la eficiencia del trabajo en equipo y se genera un modelo preliminar de relaciones.

Se recomienda que en futuros trabajos académicos se consideren las variables identificadas para que sirvan de aporte a un arquetipo de la eficiencia de equipos de trabajo. Las variables aquí identificadas se pueden cambiar o adaptar para trabajos más específicos.

\section{Referencias}

[1] C. M. Alcover, "Trabajando juntos: fundamentos de los equipos de trabajo y del trabajo en equipo", en Psicossociologia do Trabalho e das Organizacoes. Principios e Praticas, pp. 261-297, 2014. [En línea]. Disponibleen:https://www.researchgate.net/profile/ CarlosMaria_Alcover/publication/261338823_Pessoas_tarefas_processos_e_resultados_Principios_ das_equipas_de_trabalho_e_do_trabalho_em equipa/links/54e234cf0cf2c3e7d2d314ed.pdf

[2] F. A. Baldazo, M. Marcelino y M. Sánchez, "Dinámica de trabajo de las empresas familiares. Entre la conformación de grupos o equipos", Revista Multidisciplinaria de Avances de Investigación, vol. 1, n. ${ }^{\circ}$ 1, sep.-dic. 2015. [En línea]. Disponible en: http://www.remai.ipn.mx/index.php/REMAI/article/view/7 
[3] K. Puente Palacios, T. Moreira, T. Puente y N. Lira, "El consenso estratégico como predictor de resultados de equipos de trabajo", Revista de Psicología (PUCP), col. 33, no. 1, pp. 191-223, 2015. [En línea]. Disponible en: http://www.scielo.org.pe/pdf/psico/ v33n1/a07v33n1.pdf

[4] C. Torrelles, J. Coiduras, S. Isus, F. X. Carrera, G. París y M. J. Cela, "Competencia de trabajo en equipo: definición y categorización”, Profesorado, Revista de curriculum y formación del profesorado, vol. 15, no. 3, 2011. [En línea]. Disponible en: http://www. redalyc.org/pdf/567/56722230020.pdf

[5] A. R. Endara. La comunicación interna como estrategia para el fortalecimiento del trabajo en equipo en la Facultad de Ciencia e Ingeniería de Alimentos. Ambato, Ecuador: Universidad Regional Autónoma de los Andes, Facultad de Educación y Comunicación, 2015. [En línea]. Disponible en: http://186.3.45.37/ bitstream/123456789/628/1/TUAMCC024-2015. pdf

[6] M. S. Rodríguez. Empoderamiento en trabajo en equipo empresarial (ensayo). Universidad Militar Nueva Granada. Programa de Administración de Empresas, 2014. [En línea]. Disponible en: http:// unimilitar-dspace.metabiblioteca.org/bitstream/ 10654/13096/1/Ensayo\%20Sonia\%20Rodr\%C3\%ADguez.pdf

[7] M. Estrada, "Desempeño en equipos de trabajo para organizaciones cambiantes", Scientia Et Technica, vol. 16, no. 49, pp. 128-133, 2011. [En línea]. Disponible en: http://www.redalyc.org/html/849/84922625022/

[8] C. Alcover, R. Rico y F. Gil, "Equipos de trabajo en contextos organizacionales: dinámicas de cambio, adaptación y aprendizaje en entornos flexibles", Papeles del Psicólogo, pp. 7-16, 2011. [En línea]. Disponible en: http://www.redalyc.org/ html/778/77817210002/

[9] A. Martínez-Martínez, A. García-Garnica y G. Santos-Navarro, "Nuevas formas de organización laboral en la industria automotriz: lo equipos de trabajo en General Motors, Complejo Silao", Análisis Económico, vol. xxix, n. ${ }^{\circ}$ 70, pp. 157-183, 2014. [En línea]. Disponible en: http://www.redalyc.org/ html/413/41331851009/

[10] J. Martínez, "Herramienta de simulación basada en agentes para la ayuda en la formación y configuración de equipos de trabajo", tesis doctoral, Facultad de Informática, Universidad Complutense de Madrid, 2010. [En línea]. Disponible en: http://grasia. fdi.ucm.es/jpavon/tesis/jmm/PhD_MartinezMiranda_FinalVersion.pdf

[11] T. Bonavia, G. Molina y A. Puchol, "Validez estructural de un cuestionario para medir comportamientos eficaces en los equipos de trabajo", Anales de Psicolo- gía, vol. 31, no. 2, pp. 667-676, 2015. [En línea]. doi: http://dx.doi.org/10.6018/analesps.31.2.184041

[12] R. Malpica, R. Rosell y Hoffmann, "Equipos de trabajo de alto desempeño", Observatorio Laboral Revista Venezolana, vol. 7, no. 14, pp. 69-83, 2014. [En línea]. Disponible en: http://www.redalyc.org/ html/2190/219040849005/

[13] A. Blanco, "Revisión de los hallazgos de investigación sobre trabajo en equipo; revisión de cuatro grandes trabajos, tesis de grado, Facultad de Administración, Universidad del Rosario, 2013. [En línea]. Disponible en: http://repository.urosario.edu. co/handle/10336/4846

[14] S. Ayestarán, A. Aritzeta y J. Arrospide. Guía para el trabajo en equipo. Documento de la cátedra de calidad de la UPV/EHU. 2005. [En línea]. Disponible en: https://www.ehu.eus/documents/19040 00/1916168/19+Gu\%C3\%ADa+Trabajo+Equipos. pdf

[15] S. Fajardo, "Liderazgo con ética para optimizar el trabajo en equipo", tesis de grado, Facultad de Ciencias Económicas, Universidad Militar Nueva Granada, 2014. [En línea]. Disponible en: http://unimilitar-dspace.metabiblioteca.org/bitstream/10654/11437/2/ Liderazgo\%20con\%20etica\%20para\%20optimizar\%20el\%20trabajo\%20en\%20equipo.pdf

[16] E. Viles, C. Jaca, J. Campos, N. Serrano y J. Santos, "Evaluación de la competencia de trabajo en equipo en los grados de Ingeniería”, Dirección y Organización, no. 46, abr. 2012, pp. 2171-6323. [En línea]. Disponible en: http://ww.revistadyo.com/index. php/dyo/article/view/392

[17] E. Louffat. Administración: fundamentos del proceso administrativo. Argentina, Buenos Aires: Cengage Learning, 2010. [En línea]. Disponible en: https:// issuu.com/cengagelatam/docs/administracion_ proceso_administrativo_enrique_louf

[18] I. Chiavenato. Gestión del talento humano. $3^{\text {a }}$ edición, McGraw Hill, 2009. [En línea]. Disponible en: http://www.academia.edu/download/34860731/ LECTURA_CLASE1_Gestion_del_Talento_Humano.doc

[19] G. Lacalle. Las relaciones laborales en la empresa (operaciones administrativas de recursos humanos. Ciclos Formativos. 2012. [En línea]. Disponible en: https://books.google.es/books?hl=es\&lr=\&id=enr6AwAAQBAJ\&oi $=$ fnd \&pg $=$ PA55\&dq=Las + re laciones+laborales+en+la+empresa+(Operaaciones+administrativas +de+recursos +humanos\&ots $=$ agdoCptMP9\&sig $=\mathrm{jHJnUTRf5Roo-}$ cB9AFOvfXcRNnRQ\#v=onepage \&q=Las\%20 relaciones $\% 20$ laborales $\% 20$ en $\% 201$ a $\% 20$ empresa $\% 20$ (Operaaciones $\% 20$ administrativas $\% 20$ de\%20recursos\%20humanos\&f=false 
[20] N. Castro y O. Rajadel, "Sistema para el cálculo de la efectividad y la eficiencia del proceso de integración de la gestión de la ciencia, la innovación tecnológica y el medio ambiente a escala territorial", en xiv Congreso Latino-Iberoamericano de gestión tecnológica-Altec, Lima, Perú, 2011. [En línea]. Disponible en: http://www.redalyc.org/html/870/87020009002/

[21] C. M. Alcover, R. Rico y C. Tabernero, "Efectividad de los equipos de trabajo, una revisión de la última década de investigación (1999-2009)", Revista de Psicología del Trabajo y de las Organizaciones, vol. 26, no. 1, pp. 47-71, 2010. [En línea]. Disponible en: http://scielo.isciii.es/scielo.php?pi$\mathrm{d}=$ S1576-59622010000100004\&script $=$ sci_arttex$\mathrm{t} \& \mathrm{t} \operatorname{lng}=\mathrm{pt}$

[22] M. Linares-Borrell y A. Medina-León, "Procedimiento de capacitación en equipos de trabajo con enfoque de competencias. Aplicación en un equipo de alta dirección”, Ingeniería Industrial, vol. 31, no. 3, pp. 1-7, 2010. [En línea]. Disponible en: http://www. redalyc.org/html/3604/360433574006/

[23] F. J. Molina, "Construcción de equipos de trabajo mediante análisis de redes sociales e identificación de atributos personales", tesis de grado, Facultad de Ciencias Físicas y Matemáticas, Universidad de Chile, 2010. [En línea]. Disponible en: http://www.tesis. uchile.cl/tesis/uchile/2010/cf-molina_fi/pdfAmont/ cf-molina_fj.pdf

[24] N. Gamero, "El clima afectivo en equipos de trabajo: antecedentes y consecuencias", tesis doctoral, Universidad de Valencia. 2008. [En línea]. Disponible en: http://roderic.uv.es/handle/10550/15395

[25] V. Orengo, J. Peiró y A. Zornoza, “Equipos de trabajo en las organizaciones. Aportaciones recientes de la investigación y sus implicaciones para la práctica profesional", Papeles de Psicólogo, vol. 32, no. 1, pp. 2-6, 2011. [En línea]. Disponible en: http://www.redalyc.org/html/778/77817210001/

[26] H. A. Reza, "Modelo teórico, basado en la motivación, liderazgo y gestión de equipos de trabajo, para el logro de los objetivos organizacionales. Caso de estudio: subdirección comercial de una empresa de telecomunicaciones", tesis de grado, Instituto Politécnico Nacional Escuela Superior de Comercio y Administración Unidad Santo Tomas, Sección de estudios de Posgrado e Investigación, 2014. [En línea]. Disponible en: http://tesis.ipn.mx/bitstream/ handle/123456789/11833/Hector\%20Alonso\%20 Reza\%20Tapia.pdf?sequence $=1$

[27] M. Mantilla y D. García, “Trabajo en equipos autodirigidos: competencias personales y conductas necesarias para su éxito", Revista Venezolana de Gerencia, vol. 15, no. 49, pp. 51-71, 2010. [En línea]. Disponible en: http://www.redalyc.org/html/290/29012358004/
[28] J. M. Alberola, E. del Val, V. Sanchez y A. Palomares, "An artificial intelligence tool for heterogeneous team formation in the classroom", Knowledge-Based Systems, p. 14, 2016. doi: https://doi.org/10.1016/j. knosys.2016.02.010

[29] A. Portalanza, "Liderazgo distribuido en equipos de trabajo: una aproximación conceptual”, Universidad y Empresa, vol. 15, no. 25, pp. 73-85, 2013. [En línea]. Disponible en: http://www.redalyc.org/ html/1872/187229746005/

[30] E. Viles, C. Jaca, M. Tanco y A. Medina, "Desarrollo metodológico para medir el trabajo en equipo", Revista Venezonala de Gerencia, vol. 16, no. 55, pp. 375-389, 2011. [En línea]. Disponible en: http:// www.redalyc.org/html/290/29020561003/

[31] N. Hernández y P. C. Muñoz, “Trabajo colaborativo en entornos e-learning y desarrollo de competencias transversales de trabajo en equipo: análisis del caso del Master en Gestión de Proyectos en Cooperación Internacional, cseu La Salle", Revista de Docencia Universitaria, vol. 10, pp. 411-434, may.-agos. 2012. [En línea]. Disponible en: http://openaccess.uoc. edu/webapps/o2/handle/10609/26321

[32] A. F. Uribe, J. M. C. F. Molina y D. Barbosa, "Liderar equipos de alto desempeño. Un gran reto para las organizaciones actuales", Universidad y Empresa no. 24, pp. 53-71, 2013. [En línea]. Disponible en: http:// www.redalyc.org/html/1872/187229746004/

[33] F. Gil, C. M. Alcover, R. Rico y M. Sánchez, "Nuevas formas de liderazgo en equipos de trabajo", $\mathrm{Pa}$ peles de Psicólogo, vol. 32, no. 1, pp. 38-47, 2011. [En línea]. Disponible en: http://www.redalyc.org/ html/1872/187229746004/

[34] P. S. Pérez y S. Azzollini, "Liderazgo, equipos y grupos de trabajo-su relación con la satisfacción laboral", Revista de Psicología, vol. 31, no. 1, Revista de Psicología (PUCP), 2013. [En línea]. Disponible en: http://www.scielo.org.pe/scielo.php?pid=S0254-92 472013000100006\&script=sci_arttext

[35] A. Rehman, J. Jaafar, M. Omar, S. Basri y A. Waqas, "A rule-based model for software development team composition: team leader role with personality types and gender classification", Information and Software Technology, pp. 105-113, 2016. doi: https://doi. org/10.1016/j.infsof.2016.02.007

[36] M. A. Boggio, "Liderazgo en equipos de trabajo", tesis de grado, Facultad de Ciencias Económicas, Universidad Nacional de Cuyo, 2011. [En línea]. Disponible en: http://bdigital.uncu.edu.ar/objetos_digitales/4596/boggio-liderazgoenequiipostrabajo.pdf

[37] D. Haselberg, "Literature based framework of performance related leadership interactions in ICT project teams", Information and Software Technolo$g y$, vol. 70. doi: http://dx.doi.org/10.1016\%2Fj.infsof.2015.09.003 
[38] M. Turbay, "Liderazgo e innovación organizacional", Piscología desde el Caribe, vol. 30, no. 1, pp. viI-IX, 2013. [En línea]. Disponible en: http://www.redalyc. org/pdf/213/21328600001.pdf

[39] M. Benítez, F. Medina y L. Munduate, "El estudio del conflicto en los equipos de trabajo. Una visión de las contribuciones científicas realizadas en España", Papeles de psicólogo, vol. 32, no. 1, pp. 69-81, 2011. [En línea]. Disponible en: http://www.redalyc.org/ html/778/77817210008/

[40] R. E. Palamary, "Formación de equipos de alto desempeño y estrategias gerenciales en proyectos de empresas publicitarias", Estudios Gerenciales, vol. 28, no. 122 , en.-mar., pp. 69-81, 2012. doi: https://doi. org/10.1016/S0123-5923(12)70194-8

[41] M. T. Palomo, Liderazgo y motivación de equipos de trabajo. $8^{a}$ ed., 2013. [En línea]. Disponible en: https://books.google.es/books?hl=es\&lr=\&id=qEM VAwAAQBAJ\&oi $=$ fnd \&pg $=$ PA $11 \& \mathrm{dq}=$ palo mo+Liderazgo $+y+$ motivacion+de+equipos + de + trabaj\&ots=b8QC6hKu8Q\&sig=vxwXOL4NWLX$\mathrm{kQRA} 5$ pfc60BFjP-4\# $\mathrm{v}=$ onepage $\& \mathrm{q}=$ palomo $\% 20$ Liderazgo $\% 20$ y $\% 20$ motivacion $\% 20 \mathrm{de} \% 20$ equipos\%20de\%20trabaj $\& \mathrm{f}=$ false

[42] C. Margerison, D. McCann y R. Davies, "Focus on team appraisal", Team Performance Management an Internacional Journal, vol. 1, no. 4, pp. 13-18, 1995. doi: https://doi.org/10.1108/13527599510096747

[43] F. Borrell. Cómo trabajar en equipo. Ed. Gestión 2000, 2004. [En línea]. Disponible en: http://revocacionopcionesafap.bps.gub.uy/innovaportal/ file/8457/1/trabajo_en_equipo.pdf

[44] C. A. Franco, "Modelos para el análisis y el diagnóstico de los equipos de trabajo", Estudios Gerenciales, no. 91, pp. 35-48, abr.-jun. 2004. [En línea]. Disponible en: http://www.scielo.org.co/scielo.php ?pid=S0123-59232004000200002\&script=sci_art text\&tlng=pt

[45] A. A. Cuadra y C. B. Veloso, "Grado de supervisión como variable moderadora entre liderazgo y satisfacción, motivación y clima organizacional", Revista Chilena de Ingeniería, 18, pp. 15-25, 2010. doi: http://dx.doi.org/10.4067/S07183305201000010000 3.33052010000100003\&script $=$ sci_arttext\&tlng $=$ pt

[46] A. Parra y F. Gómez, "Conductas de ciudadanía organizacional y la confianza de la construcción de equipos de trabajo", Escuela Universitaria de Trabajo Social, Universidad Complutense de Madrid, 2008. [En línea]. Disponible en: https://www. researchgate.net/profile/Francisco_Gomez41/ publication/41311464_Conductas_de_Ciudadania_Organizacional_y_la_Confianza_en_la_Construccion_de_Equipos_de_Trabajo/links/00b4953b 3a77be4684000000.pdf
[47] P. Essens, A. Vogelaar, J. Miller, C. Blendell, C. Paris, S. Halpin y J. Baranski, "MIlitary command team effectiveness: model and instrument for assessment and improvement North Atlantic Treaty Organisation", Research and Technology Organisation, 2005. [En línea]. Disponible en: http://www.dtic.mil/gettr-doc/pdf?AD=ADA437898

[48] M. Bustamante, S. Llorens y H. Acosta, "Empatía y calidad de servicio: el papel clave de las emociones positivas en equipos de trabajo", Revista Latinoamericana de Psicología Positiva, vol. 1, no. 1, pp. 7-17, 2014. [En línea]. Disponible en: http://repositori.uji. es/xmlui/handle/10234/123113

[49] Bayona, J. Andrés y O. Heredia, "El concepto de equipo en la investigación sobre efectividad en equipos de trabajo", Revista de Estudios Gerenciales, vol. 28, no. 123, pp. 121-132, 2012. doi: https://doi.org/ 10.1016/S0123-5923(12)70208-5

[50] J. Navarro, S. D. Quijano, R. Berger y R. Meneses, "Grupos en las organizaciones: herramienta básica para gestionar la incertidumbre y ambigüedad crecientes", Papeles de Psicólogo, 32, pp. 17-28, 2011. [En línea]. Disponible en: http://www.redalyc.org/ $\mathrm{html} / 778 / 77817210003 /$

[51] R. Meneses y J. Navarro, “¿Cómo mejorar la eficacia de los equipos a través de los procesos grupales? Un ejemplo en la industria automotriz", Papeles del Psicólogo, vol. 36, no. 3, pp. 224-229, sept.-dic. 2015. [En línea]. Disponible en: http://www.redalyc.org/ pdf/778/77842122008.pdf

[52] J. O. Montoya-Estrada, "Metodología para medir y evaluar individualmente el trabajo en equipo", $R e$ vista Ingeniería y Sociedad, vol. 4., pp. 1-13, 2012. [En línea]. Disponible en: http://tesis.udea.edu. co/dspace/bitstream/10495/7751/1/MontoyaJaime_2012_medirevaluartrabajoequipo.pdf

[53] H. Yim y P. Seong, "A quantitative team situation awareness measurement method considering technical and nontechnical skills of teams", Nuclear Engineering and Technology, vol. 48, no. 1, pp. 144-152, feb. 2016. doi: https://doi.org/10.1016/j. net.2015.09.007

[54] P. A. Vargas y M. Osorio, "Competencia 'trabajo en equipo' en cursos de 'Práctica de Ingeniería I", El hombre y la máquina, n. ${ }^{\circ} 41$, en.-abr., 2013. Disponible en: http://www.redalyc.org/html/478/4782841 6005/

[55] D. I. González y Y. Ospina, “Trabajo en equipo en el clima organizacional de las empresas, en el área específica del talento humano", Colecciones Académicas de Ciencias Sociales, vol. 2, no. 1, 2015. [En línea]. Disponible en: http://www.ingentaconnect. com/content/doaj/24220477/2015/00000002/00000 001/art00009 
[56] Real Academia de la Lengua Española. Diccionario de la Lengua Española. RAE, 23ª ed., 2014.

[57] A. A. Álvarez y R. V. Baños, "Interpersonal truth and intercultural competence at the virtual team works", Revista Brasileña de Comunicacao Organizacional e Relacoes Publicas Organicom, vol. 11, no. 21, pp. 1-21, 2014. 